\title{
Liquid phase synthesis and sintering of $\mathrm{Y}_{3} \mathrm{NbO}_{7}$
}

\author{
S. Guené-Girard ${ }^{1 *}$, V. Jubera ${ }^{1}$, L. Viers ${ }^{2}$, R. Boulesteix ${ }^{2}$, A. Maître ${ }^{2}$ and J.-M. \\ Heintz ${ }^{1}$
}

${ }^{1}$ Univ. Bordeaux, CNRS, Bordeaux INP, ICMCB, UMR5026, F-33600 Pessac, France

${ }^{2}$ Univ. Limoges, CNRS, IRCER, UMR7315, F-87068 Limoges, France

*Corresponding author. Email address: simon.guene-girard@icmcb.cnrs.fr (S. Guené-Girard).

\section{$\underline{\text { Abstract }}$}

A new liquid phase synthesis of $\mathrm{Y}_{3} \mathrm{NbO}_{7}$ is presented. The co-precipitation method was chosen and both nitrate or chloride precursors and as well as aqueous ammonium or ammonia hydrogen carbonate as precipitant were used to obtain the cubic fluorite phase. Purity of the powders was controlled by XRD and through the luminescence of the $\mathrm{Eu}^{3+}$ ions introduced as doping element during the synthesis process. Effect of the synthesis route on particle morphology was also investigated. Then, heat treatments in air were conducted to study the sintering behavior of the material obtained from chloride precursors precipitated in ammonia. The purity of the sintered pellets was controlled. Results demonstrate the $\mathrm{Y}_{3} \mathrm{NbO}_{7}$ phase is stable even for the highest temperatures and that a relative densification of $99.6 \%$ is obtained.

\section{$\underline{\text { Keywords }}$}

$\mathrm{Y}_{3} \mathrm{NbO}_{7}$, Ceramics, Sintering, Niobate, Densification, Co-precipitation, liquid route synthesis 


\section{1) Introduction}

Rare earth niobates $\left(\mathrm{RE}_{3} \mathrm{NbO}_{7}\right)$ are materials that present interesting magnetic [1-3], dielectric [4,5], photocatalytic [6,7], electric or ionic conductivity [8-12] and optical properties $[13,14]$. Such properties could be related to the crystal structures of $\mathrm{RE}_{3} \mathrm{NbO}_{7}$ that exists in three different crystalline forms depending on the ionic radii of the rare earth atoms [3,15-19]. Orthorombic (Pnma) and cubic pyrochlore $(\mathrm{Fd} \overline{3} \mathrm{~m})$ structures are found for the largest cations, while the smallest radii $\mathrm{RE}(\mathrm{Dy}, \mathrm{Ho}, \mathrm{Er}, \mathrm{Tm}, \mathrm{Eu}, \mathrm{Yb} \ldots$ ) crystallize into a defect cubic fluorite structure $(\mathrm{Fm} \overline{3} \mathrm{~m})[15,17]$. Rare earth and niobium atoms are statistically distributed on cationic sites, as well as oxygen vacancies on anionic sites. This relative disordered structure leads to peculiar optical properties with a significant broadening of the $4 \mathrm{f}-4 \mathrm{f}$ transitions observed in an Eu-doped $\mathrm{Y}_{3} \mathrm{NbO}_{7}$ compound [13].

Because many optical applications require massive component, the implementation of these properties requires the elaboration of full densified $\mathrm{RE}_{3} \mathrm{NbO}_{7}$-based ceramics. Sintering is one of the classical methods to obtain such materials. Thus, it is possible to obtain rare earth doped YAG transparent ceramic with laser properties similar to YAG single crystal [20]. Thus, concerning the $\mathrm{RE}_{3} \mathrm{NbO}_{7}$ composition, Spark Plasma Sintering (SPS) technics was successfully used by An et al. to obtain the cubic $\mathrm{Lu}_{3} \mathrm{NbO}_{7}$ transparent ceramics [21,22]. More recently, we studied the effect of SPS process on the sintering of isostructural $\mathrm{Eu}^{3+}$-doped $\mathrm{Y}_{3} \mathrm{NbO}_{7}$ [14]. However, SPS remains quite a constraining method of densification in terms of apparatus, pressure and atmosphere, especially for optical properties. In addition, the strong reducing condition favors the reduction of $\mathrm{Nb}^{5+}$ into $\mathrm{Nb}^{4+}$, which is detrimental to a satisfying transparency in the visible range. Therefore, pressure-less sintering of rare earth niobates appears as a potential alternative method to obtain massive component. Consequently, powder preparation optimization is necessary to ensure high purity of the phase and to optimize particle morphology and grain size distribution. The synthesis of $\mathrm{Y}_{3} \mathrm{NbO}_{7}$ matrix has been reported through different experimental routes : hydrothermal [7], glycothermal [23] and Pechini method [13,14,24]. In this work, we propose an alternative 
synthesis process based on a co-precipitation method, leading to pure $\mathrm{Y}_{3} \mathrm{NbO}_{7}$ phase with a wellcontrolled particle morphology. The sintering behavior of the powder was then studied as a function of the temperature of heat treatment and dwell time.

\section{2) Materials and methods}

\section{1) Powder synthesis}

Different chemical routes have been investigated in this work to synthesize fine yttrium niobate powders $\left(\mathrm{Y}_{3} \mathrm{NbO}_{7}\right)$, showing a high sinterability. Since europium cations can substitute yttrium ions with no influence on the fluorite structure due to close radii and equal oxidation number [25], $\mathrm{Eu}^{3+}$ doping $(1 \mathrm{~mol} \%)$ has been performed in order to use the lanthanide luminescence as structural probe. For all tested synthesis routes, the precursor for $\mathrm{Nb}$ was a niobium chloride powder $\left(\mathrm{NbCl}_{5}, 99.9 \%\right.$, Alfa Aesar). Concerning rare earth elements, two different precursors were tested: yttrium/europium nitrate hydrate $\left(\mathrm{RE}\left(\mathrm{NO}_{3}\right), 6 \mathrm{H}_{2} \mathrm{O}, 99.9 \%\right.$, Alfa Aesar) and yttrium/europium chloride hydrate $\left(\mathrm{RECl}_{3}, 6 \mathrm{H}_{2} \mathrm{O}\right.$, Alfa Aesar, $99.99 \%$ purity).

Two different aqueous precursor solutions were considered: (i) the first one consisted in a solution of yttrium/europium nitrates while (ii) the second one was a solution of yttrium/europium chlorides, both dissolved in distilled water at 1.2 mol.L-1. Each solution was then mixed with a solution of niobium chloride $\left(0.4 \mathrm{~mol} . \mathrm{L}^{-1}\right)$, dissolved in hydrochloric acid $(\mathrm{HCl}, 37 \mathrm{wt} \%$, Scharlau), according to the $\mathrm{Y}_{3} \mathrm{NbO}_{7}$ stoichiometry. Chemical precipitation was conducted using a reverse-strike precipitation in order to obtain a better cations and particles size homogeneity [26]. The different cation solutions were added dropwise to a precipitant solution under continuous stirring. Two different basic aqueous precipitating solutions were also considered: Ammonia, (A, Fisher Chemicals) and Ammonia Hydrogen Carbonate (AHC, Sigma Aldrich) giving rise to four different synthesis routes. In the case of aqueous ammonia, the $\mathrm{pH}$ value was maintained to 9 during all the process by addition of supplementary ammonia. In the case of ammonia hydrogen carbonate, a solution in $20 \%$ molar excess of AHC was used in order to insure 
the full precipitation of the precursors as well as the neutralization of the excess $\mathrm{HCl}$. After precipitation of all the precursors, the suspension obtained was aged during 3 days at room temperature under continuous stirring. The obtained amorphous product was recovered and washed by centrifugation several times, using successively distilled water and absolute ethanol. Once dried, the precipitate was grinded in a mortar and calcined in air at different temperatures. Four different powders were finally prepared. Table 1 provides the synthesis parameters and designations of these powders.

Table 1. Sample designations according to cation precursors and precipitant

\begin{tabular}{llll}
\hline Designation & Precursors & & Precipitant \\
\hline N-A & $\mathrm{Y}, \mathrm{Eu}\left(\mathrm{NO}_{3}\right) \cdot 6 \mathrm{H}_{2} \mathrm{O}$ & $\mathrm{NbCl}_{5}$ & $\mathrm{NH}_{4} \mathrm{OH}$ \\
\hline N-AHC & $\mathrm{Y}, \mathrm{Eu}\left(\mathrm{NO}_{3}\right) \cdot 6 \mathrm{H}_{2} \mathrm{O}$ & $\mathrm{NbCl}_{5}$ & $\mathrm{NH}_{4} \mathrm{HCO}_{3}$ \\
\hline Cl-A & $\mathrm{Y}, \mathrm{EuCl}_{3} \cdot 6 \mathrm{H}_{2} \mathrm{O}$ & $\mathrm{NbCl}_{5}$ & $\mathrm{NH}_{4} \mathrm{OH}$ \\
\hline Cl-AHC & $\mathrm{Y}, \mathrm{EuCl}_{3} \cdot 6 \mathrm{H}_{2} \mathrm{O}$ & $\mathrm{NbCl}_{5}$ & $\mathrm{NH}_{4} \mathrm{HCO}_{3}$ \\
\hline
\end{tabular}

Also, $\mathrm{Eu}: \mathrm{YNbO}_{4}$ and $\mathrm{Eu}: \mathrm{Y}_{2} \mathrm{O}_{3}$ powders were synthetized by similar chemical route. Such phases are expected to be found as impurities in the desired $\mathrm{Y}_{3} \mathrm{NbO}_{7}$ phase. Thus $\mathrm{Eu}^{3+}$ photoluminescence will be measured on these compounds and will be used as standards to verify the purity of $\mathrm{Eu}: \mathrm{Y}_{3} \mathrm{NbO}_{7}(1 \% \mathrm{~mol})$ material. $\mathrm{Eu}: \mathrm{YNbO}_{4}(1 \% \mathrm{~mol})$ was prepared with the $\mathrm{Cl}-\mathrm{A}$ route, by changing the cationic ratio. Eu: $\mathrm{Y}_{2} \mathrm{O}_{3}(1 \% \mathrm{~mol})$ was prepared by solid mixing of yttrium and europium nitrate and calcination at $1100{ }^{\circ} \mathrm{C}$.

\section{2) Sintering process}

The sintering study was carried out only for the purest powder, obtained through the chloride/ammonia process (Cl-A powder). This $\mathrm{Cl}$-A crystallized powder was planetary ball milled, sieved through a $100 \mu \mathrm{m}$ mesh and pressed into green pellets by uniaxial pressing at room temperature (applied stress equal to $125 \mathrm{MPa}$ ). Sintering experiments were performed in air, in a 
muffle furnace, up to $1700{ }^{\circ} \mathrm{C}$ with a heating rate of $10{ }^{\circ} \mathrm{C} / \mathrm{min}$. The sintering temperature varied from $1400{ }^{\circ} \mathrm{C}$ to $1650{ }^{\circ} \mathrm{C}$ and the sintering time from $1 \mathrm{~h}$ to $24 \mathrm{~h}$.

\section{3) Characterizations}

Powders and pellets were analyzed at room temperature using X-ray diffraction technique (XRD). Diffraction patterns were collected on a PANalytical X'Pert MDP-PRO X-ray diffractometer with a Bragg-Brentano $\theta-\theta$ geometry using $\mathrm{Cu} \mathrm{K} \alpha 1,2$ radiation $\left(8^{\circ}<2 \theta<80^{\circ}\right)$, with a step size of $0.02^{\circ}$ and a counting time of $30 \mathrm{~s}$. The photoluminescence properties of the Eu-doped powders and pellets were analyzed using a Horiba Fluorolog 3 spectrofluorimeter. The emission spectra were corrected for the transmission of the monochromator and the response of the photomultiplier. The equipment used consisted of a $450 \mathrm{~W}$ xenon lamp, an excitation double monochromator, an emission double monochromator and a thermo-electrically cooled photomultiplier tube.

The morphology of the powders was observed by scanning electron microscopy (TESCAN Vega II SBH SEM). Granulometry measurements were realized on a Pratica LA-950V2 apparatus from Horiba and the specific surface area was obtained by BET approximation (Brunauer-EmmettTeller theory, 3Flex Micrometrics under $\mathrm{N}_{2}$ ).

Shrinkage evolution of the powder was determined by dilatometric measurements, using a thermomechanical analyzer SETSYS Evolution TMA (SETARAM instrumentation). The pellet was firstly pressed under an uniaxial applied at $125 \mathrm{MPa}$ at room temperature and then heated up to $1700{ }^{\circ} \mathrm{C}$ with a heating rate of $10{ }^{\circ} \mathrm{C} / \mathrm{min}$ under flowing air. Green density of the pellets was determined from geometric measurements while sintered density was measured by Archimede's method in diethyl phthalate. These values are expressed as relative densities $\left(\mathrm{d}_{\mathrm{rel}}\right)$ corresponding to the density of the pellets divided by the theoretical density of Eu- $\mathrm{Y}_{3} \mathrm{NbO}_{7}$, taken as $\mathrm{d}_{\mathrm{th}}=5.436$ g.cm ${ }^{-3}\left(\right.$ JCPDS $\mathrm{N}^{\circ}$ 01-074-6421). Grain size measurements were performed on SEM micrographs of fractured samples for sintering temperature below $1525{ }^{\circ} \mathrm{C}$ and using the intercept 
method (on 3 SEM micrographs) on a polished section after thermal etching for temperatures higher than $1525^{\circ} \mathrm{C}$.

\section{3) Results and discussion}

\section{1) Role of the precipitant and the precursors}

The diffraction patterns of the washed and dried precipitates are shown in Fig.1. The nature of the precipitant has a strong impact on the structure of the powder. The dried powders synthetized with ammonia solution are mainly amorphous, whereas synthetized powders with AHC show diffraction peaks that can be attributed to different phases of yttrium oxychloride, carbonate and hydroxide and niobium oxychloride. Numerous peaks were not attributed and can be related to nitrate, ammonium, carbonate and chloride sub-products from the different reactants. As the washing processes were the same for all routes, we concluded the observed species result from reaction between reactants.

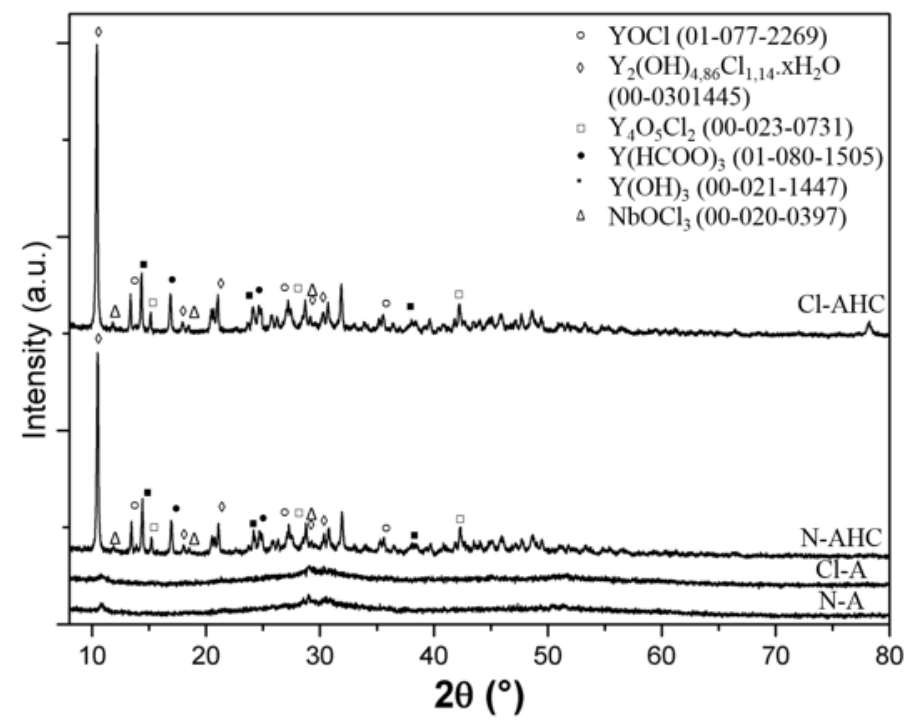

Fig.1. XRD patterns of the as washed and dried powders.

For example, the TGA curve of Cl-A powder reveals the elimination of several species, all along the temperature increase (Fig.2). As expected water and $\mathrm{OH}$ groups are decomposed up to $200{ }^{\circ} \mathrm{C}$, followed by the decomposition of nitrate compounds up to $700{ }^{\circ} \mathrm{C}$ and finally elimination of 
chlorides group at higher temperatures. A minimum calcination temperature of $900{ }^{\circ} \mathrm{C}$ was therefore used, as it allowed elimination of the majority of the synthesis' residues.

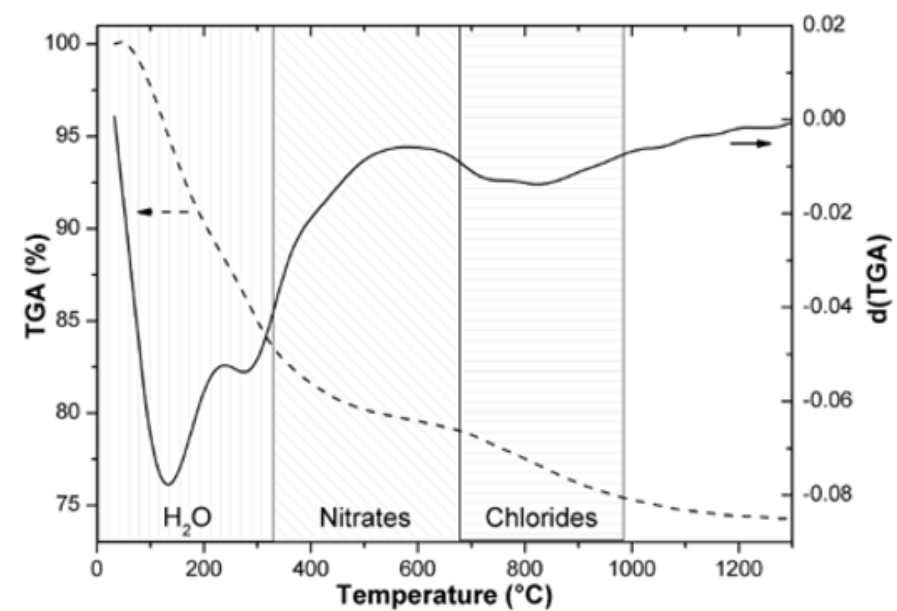

Fig.2. TGA curve of the Cl-A powder.

The dried precipitates were calcined at $900,1100,1400{ }^{\circ} \mathrm{C}$ to study the formation of the $\mathrm{Y}_{3} \mathrm{NbO}_{7}$ phase and its stability versus temperature. XRD patterns are presented in Fig.3. Heat treatment conducted at $900{ }^{\circ} \mathrm{C}$ was sufficient to observe the diffraction peaks of the expected compound. But additional contributions are detected for the AHC route. Indeed, powders obtained from the AHC route (N-AHC and Cl-AHC) are composed of three phases, $\mathrm{Y}_{3} \mathrm{NbO}_{7}$ (JCPDS N ${ }^{\circ}$ 01-0746421), $\mathrm{Y}_{2} \mathrm{O}_{3}\left(\mathrm{JCPDS} \mathrm{N}^{\circ} 88-2168\right)$ and $\mathrm{YNbO}_{4}\left(\mathrm{JCPDS} \mathrm{N}^{\circ}\right.$ 72-2077). The presence of other phases than the fluorite one can be explained by a poor cation homogeneity due to the presence of the carbonate groups. YAG synthesis mechanism' by co-precipitation has been previously described in the literature [27], we propose a similar process. We suggest that the formation of the niobate phase may occur by precipitation of $\mathrm{Y}(\mathrm{OH})_{3}$ particles followed by the diffusion of niobium ions into those particles. The carbonate groups might be an obstacle for the diffusion of niobium ions, explaining the poor homogeneity of the AHC co-precipitated powders. Furthermore, an increase of the temperature results in the disappearance of the $\mathrm{Y}_{2} \mathrm{O}_{3}$ sesquioxide. This could be explained by the reaction between $\mathrm{Y}_{2} \mathrm{O}_{3}$ and amorphous niobium compounds, leading to the increase of $\mathrm{Y}_{3} \mathrm{NbO}_{7}$ phase proportion via homogenization of cations by diffusion at high temperature. However, small diffractions peaks relative to $\mathrm{YNbO}_{4}$ are still present at that temperature. Another 
hypothesis may be based on the fluorite domain of existence. The $\mathrm{Y}_{3} \mathrm{NbO}_{7}$ composition belongs to a solid solution domain in which the cationic ratio $\mathrm{Nb} /(\mathrm{Y}+\mathrm{Nb})$ varies from about $21 \%$ to $28 \%$ [28,29]. The decrease of the $\mathrm{Y}_{2} \mathrm{O}_{3}$ amount in the solution may also reflect an enrichment of the cubic solid solution in yttrium leading to a cationic ratio inferior to the targeted $25 \%$. The modification of the fluorite composition may occur with precipitation of the $\mathrm{YNbO}_{4}$ monoclinic phase.

The precipitates obtained with aqueous ammonia ( $\mathrm{N}-\mathrm{A}$ and $\mathrm{Cl}-\mathrm{A})$ show diffraction peaks consistent with the $\mathrm{Y}_{3} \mathrm{NbO}_{7}$ fluorine type structure, up to $1100{ }^{\circ} \mathrm{C}$ for both cation precursors (Fig.3 (a) and (b)). However, at $1400{ }^{\circ} \mathrm{C}$, the N-A powder XRD pattern shows the presence of $\mathrm{YNbO}_{4}$ peaks. This could be attributed to a partial decomposition of the fluorite phase into the monoclinic one, as it has already been observed for heat treatment of $\mathrm{Y}_{3} \mathrm{NbO}_{7}$ powders obtained from Pechini chemical routes [30].
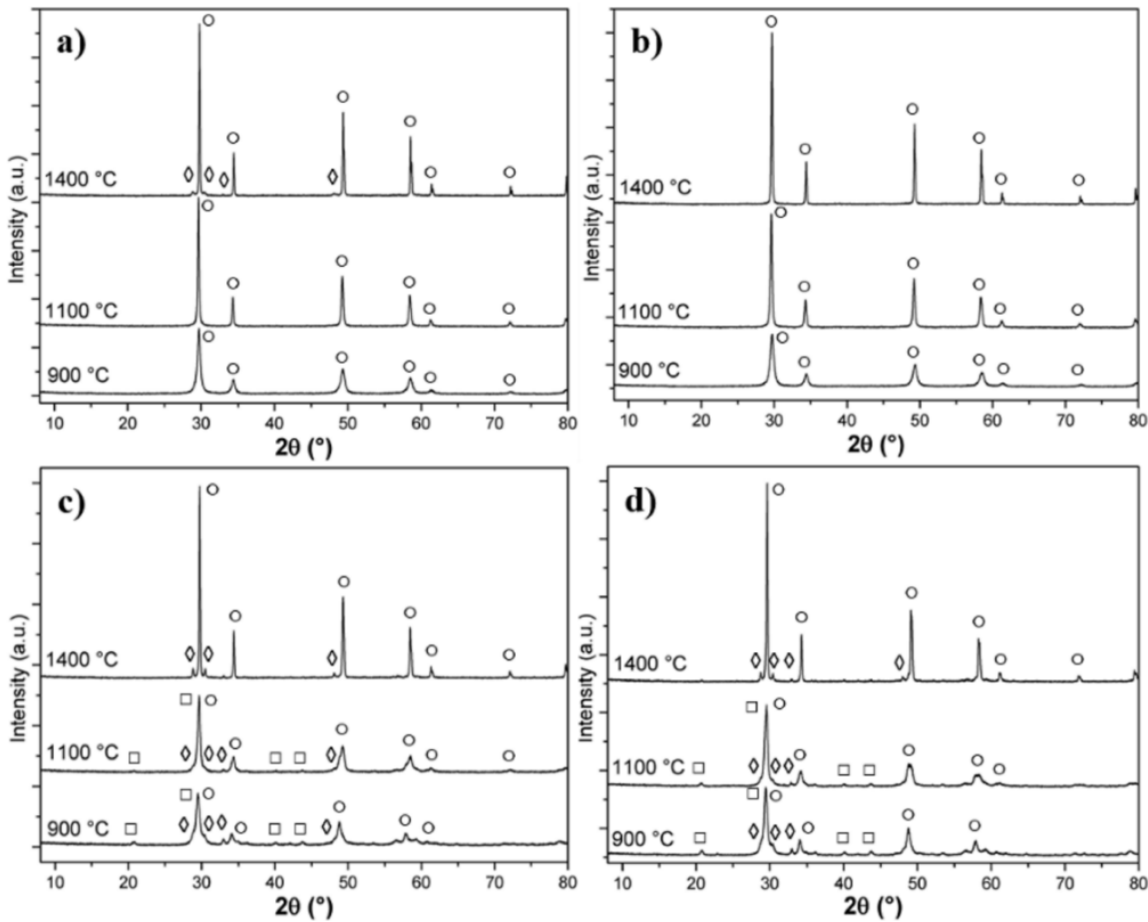

Fig.3. XRD patterns of a) $\mathrm{N}-\mathrm{A}, \mathrm{b}) \mathrm{Cl}-\mathrm{A}, \mathrm{c}) \mathrm{N}-\mathrm{AHC}$, d) Cl-AHC powders, heat-treated at different temperature ( $\mathrm{Y}_{3} \mathrm{NbO}_{7}, \mathrm{JCPDS} \mathrm{N}^{\circ}$ 01-074-6421; $\mathrm{YNbO}_{4}, \mathrm{JCPDS} \mathrm{N}^{\circ}$ 72-2077; $\mathrm{Y}_{2} \mathrm{O}_{3}, \mathrm{JCPDS}$ $\left.N^{\circ} 88-2168\right)$ 
The $\mathrm{Cl}-\mathrm{A}$ powder synthesis route seems to be the most appropriate process to stabilize a pure and stable $\mathrm{Y}_{3} \mathrm{NbO}_{7}$ powder according to these first XRD analyses. Because of its high sensitivity, photoluminescence of $\mathrm{Eu}^{3+}$ was then used to check the absence of secondary phases. Emission spectra of the $\mathrm{Cl}-\mathrm{A}$ powder calcined at $1100{ }^{\circ} \mathrm{C}$ and of two main secondary phases that can be expected in Y-Nb-O ternary system are presented in Fig.4. As already shown by Kim et al.[13], $\mathrm{Y}_{2} \mathrm{O}_{3}: \mathrm{Eu}$ and $\mathrm{YNbO}_{4}:$ Eu present sharp maximum emission lines for the hypersensitive ${ }^{5} \mathrm{D}_{0} \rightarrow{ }^{7} \mathrm{~F}_{2}$ electric dipole transition, whereas $\mathrm{Eu}^{3+}$ ions present in the fluorite phase show large response due to the intrinsic disorder of this structure. By consequence, the spectral responses of the three phases can be easily discriminated and the photoluminescence can be applied as an efficient probe to validate the purity of the powder after synthesis. Since no sharp luminescence peaks have been detected in the $600-620 \mathrm{~nm}$ range corresponding to the energy of the hypersensitive transition mentioned above, we can conclude that the $\mathrm{Cl}$-A powder is definitely pure without any secondary phase.

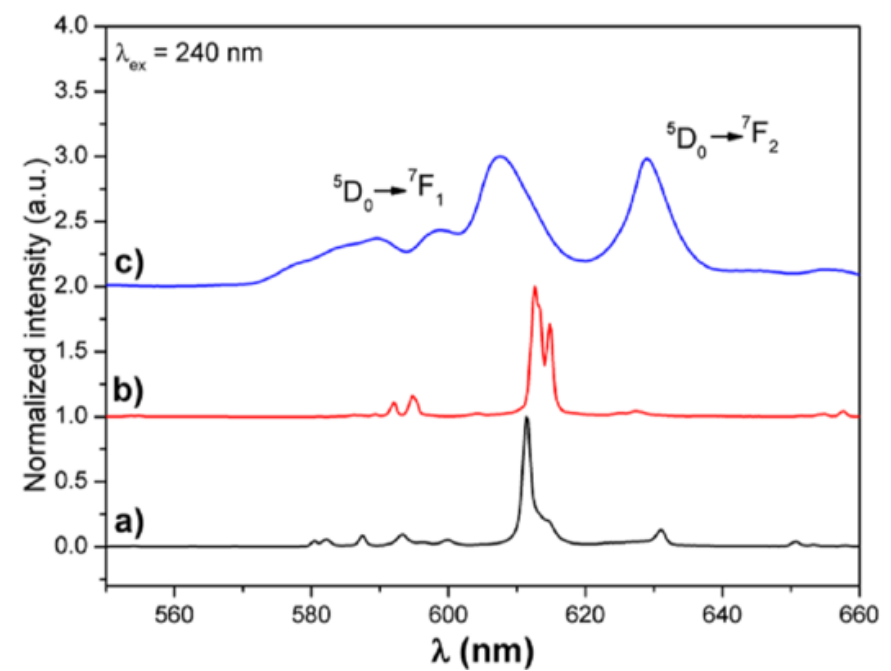

Fig.4. Photoluminescence spectra of a) $\left.\mathrm{Y}_{2} \mathrm{O}_{3}: E u, b\right) \mathrm{YNbO}_{4}: E u$, and c) $\mathrm{Cl}-\mathrm{A} \mathrm{Y}_{3} \mathrm{NbO}_{7}: E u$ powder calcined at $1100^{\circ} \mathrm{C}$.

The morphology of the powders calcined at $1100{ }^{\circ} \mathrm{C}$ is presented in Fig.5. Significant variations in morphology can be observed depending on the chosen synthesis route. Firstly, nitrate precursor (N-A and N-AHC) leads to a weakly aggregated powder, with almost individual particles. 
Whereas chloride precursor ( $\mathrm{Cl}-\mathrm{A}$ and $\mathrm{Cl}-\mathrm{AHC})$ provides a more aggregated powder with microsized aggregates. Secondly, particles which precipitated by aqueous ammonia ( $\mathrm{N}-\mathrm{A}$ and $\mathrm{Cl}-$ A) present a spherical morphology, similar in size for both precursors. Conversely, powders synthetized using AHC as precipitant display various morphologies and sizes. Fine nonaggregated particles are present as well as flakes. This combination of morphologies can be related to the XRD phase analysis that shows for this precipitant, the presence of three different phases, such as $\mathrm{Y}_{3} \mathrm{NbO}_{7}, \mathrm{Y}_{2} \mathrm{O}_{3}$ and $\mathrm{YNbO}_{4}$. Similar morphologies have been observed in the literature relative to the preparation of YAG powders using AHC as a precipitant and for high AHC concentration [31]. In this case, yttrium hydroxide precipitates as micrometer flake-shaped particles. Such large particles lead then to an increase of the diffusion distance needed to homogenize yttrium and niobium atoms, explaining the presence of several phases in the powder.

Fig.5. SEM micrographs of a) N-A, b) Cl-A, c) N-AHC, d) Cl-AHC powders, calcined at
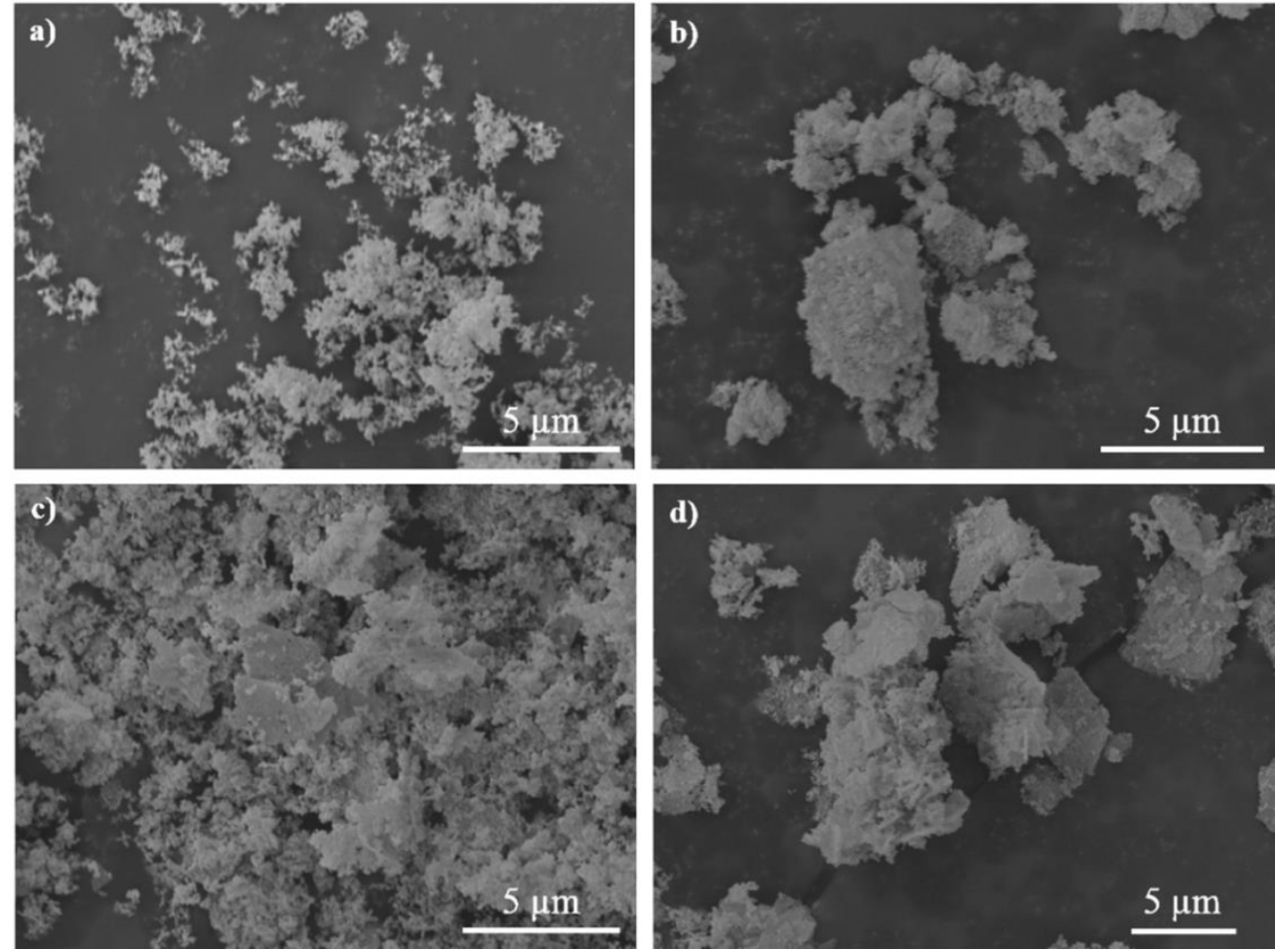

$1100{ }^{\circ} \mathrm{C}$

From a morphological point of view, the most interesting powder for sintering is the one resulting from the nitrate precursor and precipitated with ammonia. Indeed, in this latter case, 
particles appear non agglomerated and spherical. However, XRD showed that this N-A powder was not pure at high temperature. Therefore, only the monophasic $\mathrm{Cl}-\mathrm{A}$ powder that is stable at high temperature was studied in the following.

\section{2) Study of the sintering behavior of the $\mathrm{Cl}-\mathrm{A}$ powder}

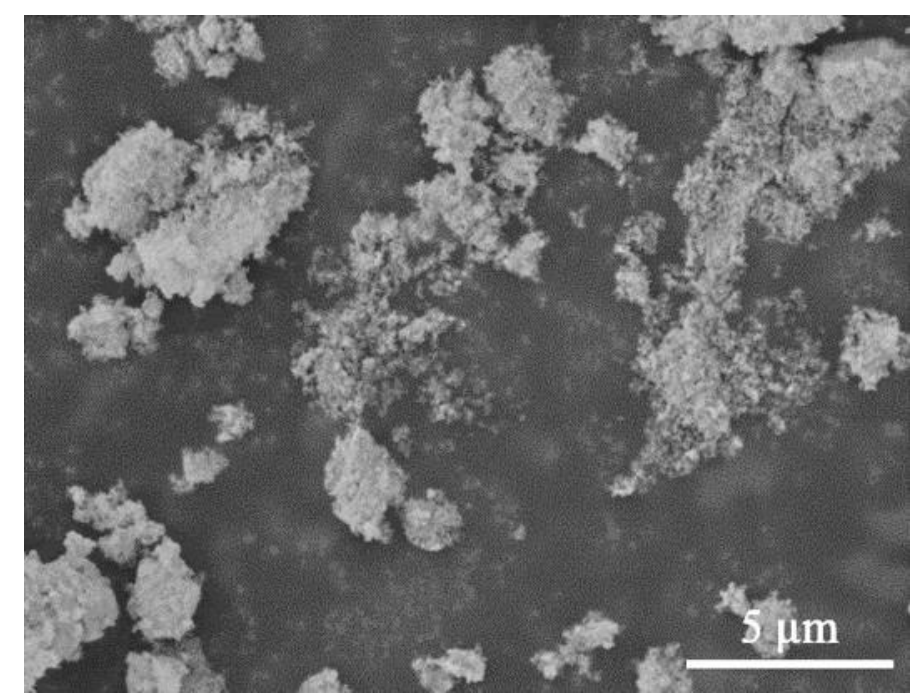

Fig.6. SEM micrograph of the calcined Cl-A powder after ball milling.

As the morphology was not fully satisfying for sintering, the Cl-A powder was planetary ball milled after calcination at $1100{ }^{\circ} \mathrm{C}$. SEM micrograph (Fig. 6) shows the effective disaggregation of many agglomerates initially present in this powder. BET measurements gave a specific surface area of $17.1 \mathrm{~m}^{2} \cdot \mathrm{g}^{-1}$. A BET equivalent particle size can be deduced from the surface area according to the following formula:

$$
\mathrm{d}_{\mathrm{BET}}=\frac{\mathrm{k}}{\rho * S S A}
$$

where $\mathrm{k}$, is a shape factor taken here as 6 assuming that particles are spherical, $\rho$ the theoretical density $\left(5.436 \mathrm{~g} . \mathrm{cm}^{-3}\right)$ and SSA the Specific Surface Area.

Under these conditions, a particle size of $d_{\text {BET }}=68 \mathrm{~nm}$ can be obtained. This result is quite consistent with the values of the average elementary particle size estimated from both image analysis of particles on SEM micrographs $(60 \pm 20 \mathrm{~nm})$ and granulometry measurements $(70 \mathrm{~nm})$. This means that the particles of this powder can be considered almost spherical and weakly 
agglomerated, resulting in a high green relative density value (51\%), considering the small grain size (less than $100 \mathrm{~nm}$ ) and the compression method (dry and uniaxial).

The sintering curve of $\mathrm{Cl}-\mathrm{A}$ sample is reported up to $1700{ }^{\circ} \mathrm{C}$ in Fig.7. The shrinkage starts at around $1200{ }^{\circ} \mathrm{C}$. It also indicates that the initial niobate powder must not be calcined at temperatures higher than $1100{ }^{\circ} \mathrm{C}$ in order to avoid formation of hard aggregates that could not be released by ball milling. The shrinkage rate increases rapidly above $1200{ }^{\circ} \mathrm{C}$ and two steps can be observed. The first one, around $1500^{\circ} \mathrm{C}$, could be likely related to the densification within some more packed zones of the granular compact, those zones can results of remaining agglomerates that can be seen Fig. 6. The second step consist of the final densification of the pellet with a maximum of the shrinkage rate observed at $1650{ }^{\circ} \mathrm{C}$. The final relative density of that sample sintered at $1700{ }^{\circ} \mathrm{C}$ was measured to be equal to $94.5 \%$ which shows the pretty good densification ability of this powder, considering that $\mathrm{Y}_{3} \mathrm{NbO}_{7}$ phase is a refractory material that needs high temperatures to be fully densified.

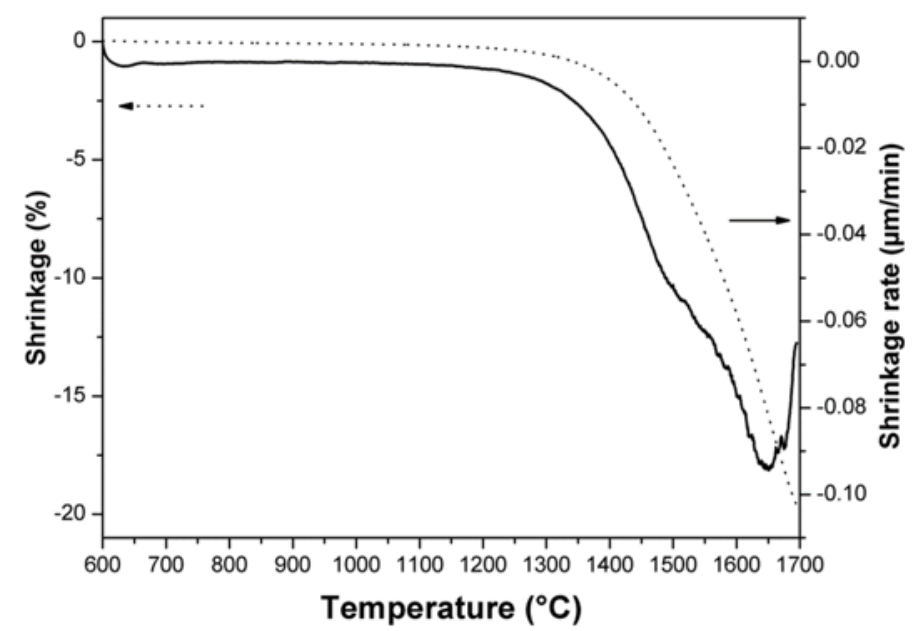

Fig.7. Dilatometric curve of the $\mathrm{Cl}$-A powder calcined at $1100^{\circ} \mathrm{C}$, ball milled and uniaxially pressed at $125 \mathrm{MPa}$.

The study of the isothermal sintering of the Cl-A powder at $1600{ }^{\circ} \mathrm{C}$ is presented in Fig.8. High relative densities are obtained from $1 \mathrm{~h}$ of treatment (Fig.8(a), $\mathrm{d}_{\text {rel }}>97 \%$ ), confirming the good sinterability of this powder. The highest density is observed for a $6 \mathrm{~h}$ heat treatment: $\mathrm{d}_{\mathrm{rel}}=99.6 \%$. Grain growth is noticeable in these conditions, with an average grain size higher than $1 \mu \mathrm{m}$ after 
a $1 \mathrm{~h}$ sintering treatment. Naming $\mathrm{G}$ the grain size at a given temperature and $\mathrm{G}_{0}$ the initial particle size, a classical $\mathrm{G}^{\mathrm{m}}-\mathrm{G}_{\mathrm{o}}{ }^{\mathrm{m}}$ law with $\mathrm{m}=3$ is observed for the last stage of sintering, up to $12 \mathrm{~h}$ of sintering (Fig.8(b)). It can be related to a pore control mechanism, trough lattice diffusion, considering that the niobate phase is pure [32,33].

Fig.8. Influence of the sintering time at $1600{ }^{\circ} \mathrm{C}$ on a) relative density and b) grain size of sample Cl-A (insert shows the linear evaluation of $G^{3}-G_{o}{ }^{3}=f(t)$ ).
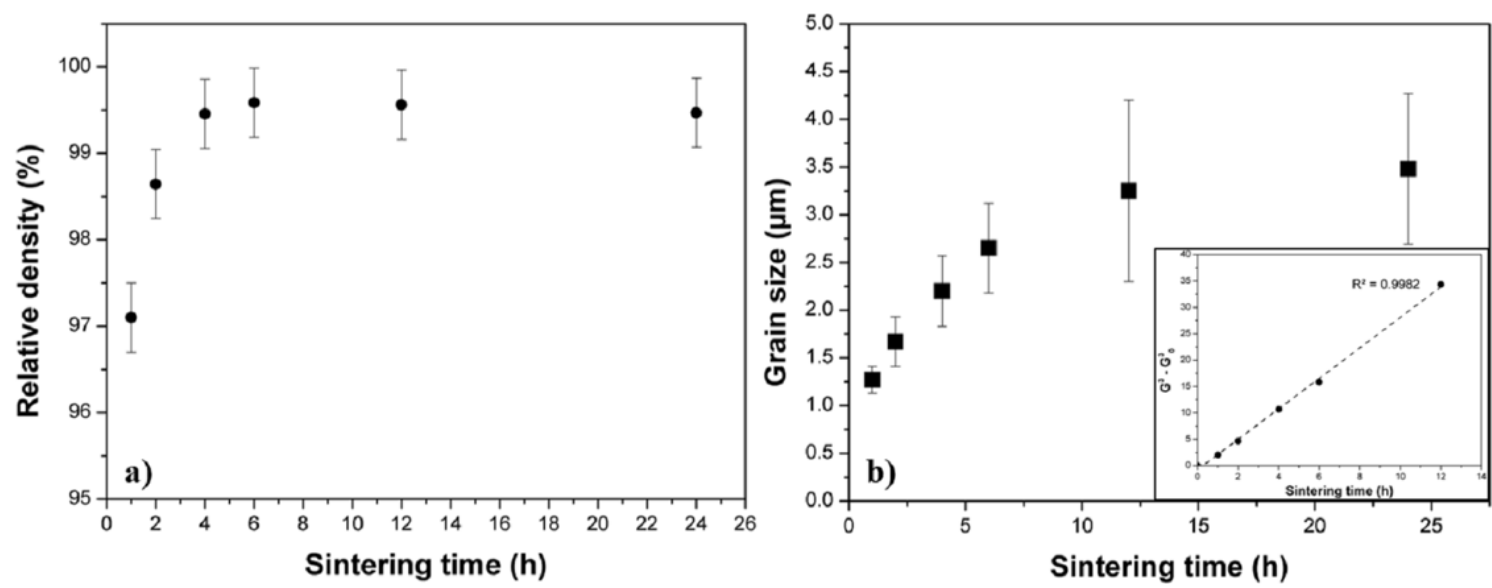

To complete this study, the influence of the sintering temperature was studied for a $6 \mathrm{~h}$ dwell time

(Fig.9). The maximum of densification is reached at $1600{ }^{\circ} \mathrm{C}$ with $\mathrm{d}_{\mathrm{rel}}=99.6 \%$ and the relative density remains stable up to $24 \mathrm{~h}$ (Fig.9(a)). The evolution of the microstructure of the sintered $\mathrm{Cl}-\mathrm{A}$ pellets as a function of the temperature is presented in Fig. 10 . From $1400{ }^{\circ} \mathrm{C}$, a very homogeneous microstructure can be observed with a regular distribution of porosity and a significant growth compared to the initial powder. At $1550{ }^{\circ} \mathrm{C}$ (Fig. $\left.10(\mathrm{c})\right)$, the third stage of sintering is reached with only closed porosity and microsized grains. At $1600{ }^{\circ} \mathrm{C}$ (Fig.10(d)), very few pores remain in the ceramic and the grains are homogeneous in size, around $2.6 \mu \mathrm{m}$. Grain growth evolution as a function of temperature is presented in Fig.9(b). A clear increase in size is noticed above $1550{ }^{\circ} \mathrm{C}$, which is consistent with SEM micrographs (Fig.10) and with achieving a final density close to $100 \%$. 

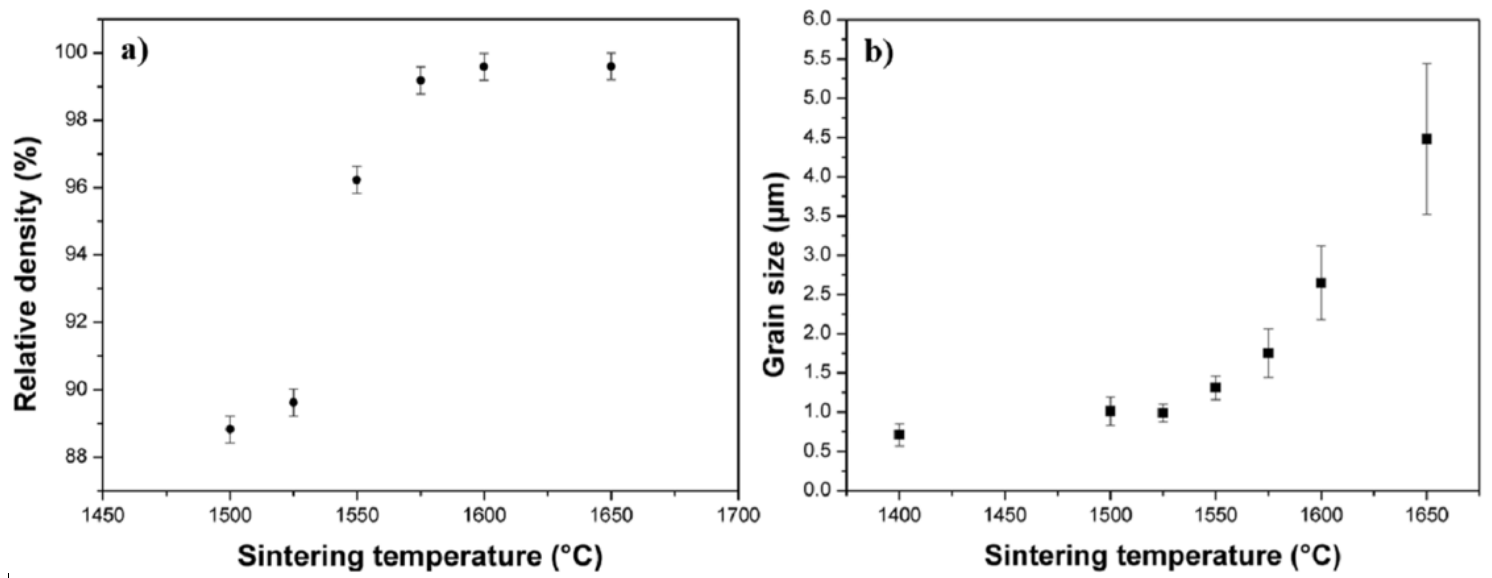

Fig.9. Evolution of a) the relative density and b) grain growth evolution of sample Cl-A as a function of the sintering temperature (dwell time $=6 \mathrm{~h}$ ).
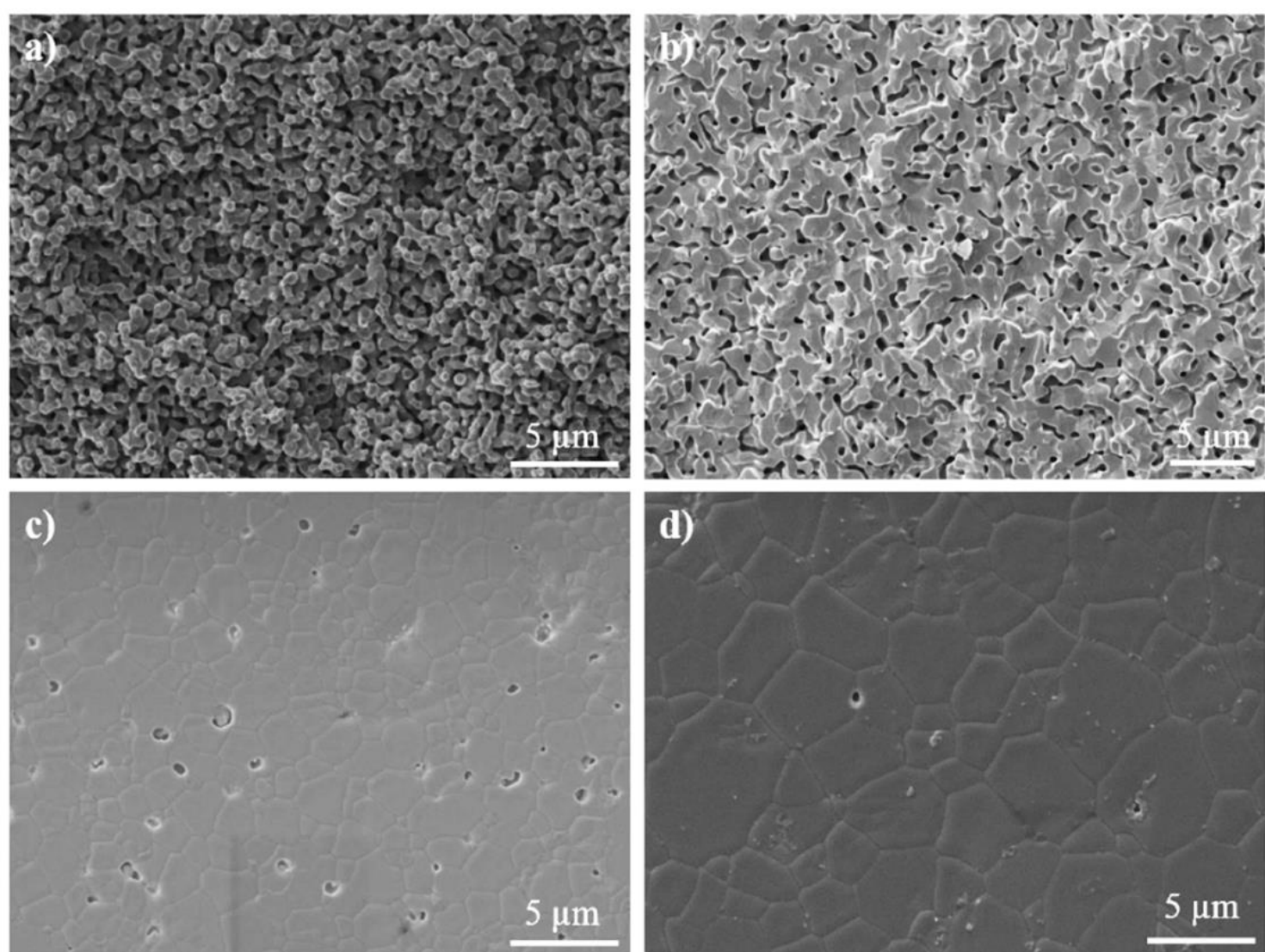

Fig.10. SEM micrographs of pellets sintered 6 h at a) $1400{ }^{\circ} \mathrm{C}$ (fracture), b) $1500{ }^{\circ} \mathrm{C}$ (fracture),

c) $1550^{\circ} \mathrm{C}$ (thermally etched surface), and d) $1600{ }^{\circ} \mathrm{C}$ (thermally etched surface).

The representation of all these experiments can be summarized on a sintering map (Fig.11). A unique trajectory is obtained, showing a clear predominance of densification over grain growth up to a relative density of $99 \%$, meaning that full density can be achieved with our powder. 
X-ray diffraction pattern of a pellet sintered at $1600{ }^{\circ} \mathrm{C}$ for $6 \mathrm{~h}$ and the corresponding photoluminescence spectrum are presented in Fig.12. No diffraction peaks of secondary phases can be observed on the XRD analysis. In addition, the photoluminescence spectrum shows a unique spectral repartition which is characteristic of the $\mathrm{Eu}^{3+}$-doped $\mathrm{Y}_{3} \mathrm{NbO}_{7}$. The $\mathrm{Y}_{3} \mathrm{NbO}_{7}$ ceramic, synthesized by the ammonia precipitation method (Cl-A) and sintered at $1600{ }^{\circ} \mathrm{C}$ is not only dense but is also stable at high temperature, showing no phase decomposition.

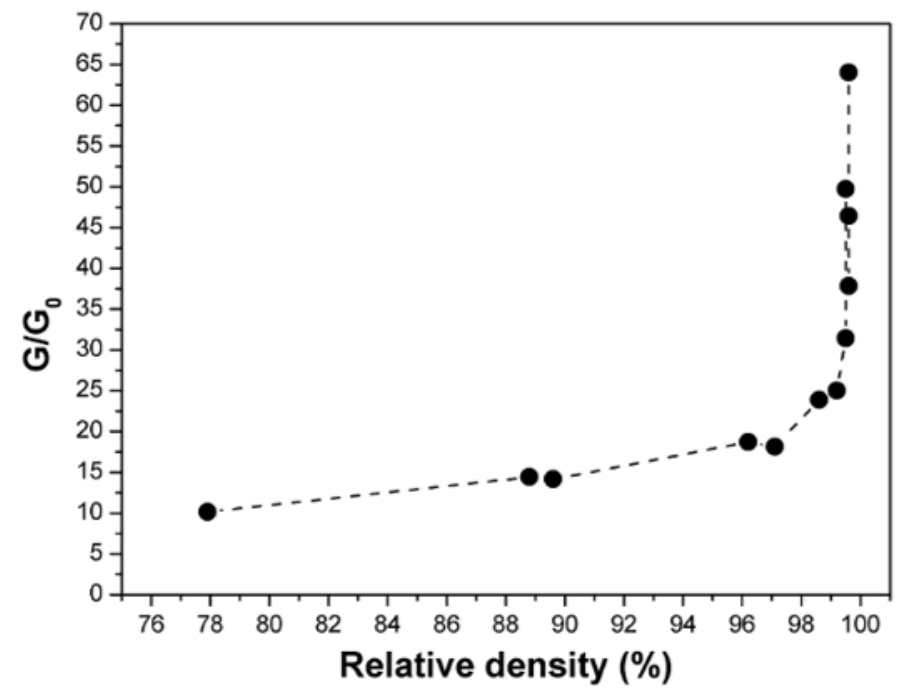

Fig.11. Sintering map of sample Cl-A.

The best density achieved by natural sintering of this $\mathrm{Cl}$-A powder is $\mathrm{d}_{\mathrm{rel}}=99,6 \%$, for a $1600{ }^{\circ} \mathrm{C}$ heat treatment during $6 \mathrm{~h}$. It shows that the composition as well as the particles morphology of the initial powder were well controlled. Considering that this powder has a high sintering potential (see the sintering map), some improvements could likely be made, in order to reach full density $\left(\mathrm{d}_{\mathrm{rel}}=100 \%\right)$. For example, the initial packing of the powder before sintering can be improved using slip casting technics or the densification itself with the use of alternative sintering technics like vacuum sintering or high isostatic pressure sintering. 

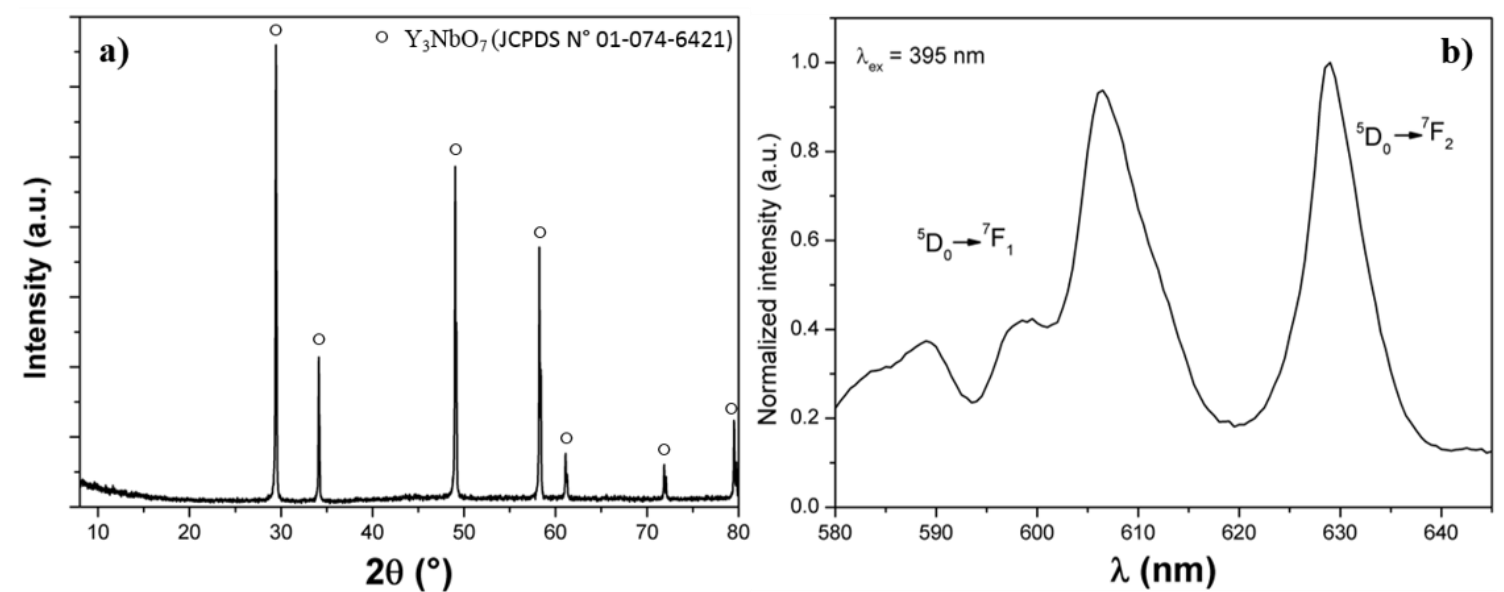

Fig.12. a) XRD pattern and b) photoluminescence spectrum of a pellet sintered $6 \mathrm{~h}$ at $1600{ }^{\circ} \mathrm{C}$.

\section{4) Conclusions}

The synthesis of a pure $\mathrm{Y}_{3} \mathrm{NbO}_{7}$ powder, presenting a high sinterability was investigated through a liquid phase synthesis. A co-precipitation route was developed using chloride precursors and aqueous ammonia as precipitant. The synthetized product was calcined at $1100{ }^{\circ} \mathrm{C}$. Crystallization of a pure cubic fluorite phase was validated by XRD and the $\mathrm{Eu}^{3+}$ spectral distribution. Preparation of the powder by ball-milling leads to high green densities and an excellent sinterability: a $99.6 \%$ relative density was measured on a pellet sintered in air at $1600{ }^{\circ} \mathrm{C}$ during $6 \mathrm{~h}$. The sintering map indicates that a full densification could be reached. Effort on green preparation and alternative sintering technics will have to be performed in order to reach full densification and transparency.

\section{Acknowledgments}

The authors thank the CNRS, the Region Nouvelle-Aquitaine. This study was carried out with financial support of the Region Nouvelle-Aquitaine (MISTRAL program $n^{\circ}$ 2017-1R50311) in the frame of "the Investments for the future" Program IdEx Bordeaux - LAPHIA (ANR-10IDEX-03-02) 


\section{$\underline{\text { References }}$}

[1] M. Wakeshima, Y. Hinatsu, Magnetic properties and structural transitions of orthorhombic fluoriterelated compounds Ln3MO7 ( $\mathrm{Ln}=$ rare earths, $\mathrm{M}=$ transition metals), Journal of Solid State Chemistry. 183 (2010) 2681-2688. https://doi.org/10.1016/j.jssc.2010.09.005.

[2] J.F. Vente, R.B. Helmholdt, D.J.W. IJdo, The Structure and Magnetic Properties of Pr3MO7 with M= $\mathrm{Nb}, \mathrm{Ta}$, and Sb, Journal of Solid State Chemistry. 108 (1994) 18-23. https://doi.org/10.1006/jssc.1994.1003.

[3] Y. Doi, Y. Harada, Y. Hinatsu, Crystal structures and magnetic properties of fluorite-related oxides Ln3NbO7 (Ln=lanthanides), Journal of Solid State Chemistry. 182 (2009) 709-715. https://doi.org/10.1016/j.jssc.2008.12.012.

[4] L. Cai, J.C. Nino, Phase formation and dielectric properties of $\operatorname{Ln} 2\left(\operatorname{Ln}^{\prime} 0.5 \mathrm{Nb} 0.5\right) 207$ ( $\mathrm{Ln}=$ rare earth element), Journal of the European Ceramic Society. 30 (2010) 307-313. https://doi.org/10.1016/j.jeurceramsoc.2009.05.026.

[5] L. Cai, J.C. Nino, Structure and dielectric properties of $\mathrm{Ln} 3 \mathrm{NbO}$ ( $\mathrm{Ln}=\mathrm{Nd}, \mathrm{Gd}, \mathrm{Dy}, \mathrm{Er}, \mathrm{Yb}$ and $\mathrm{Y}$ ), Journal of the European Ceramic Society. 27 (2007) 3971-3976. https://doi.org/10.1016/j.jeurceramsoc.2007.02.077.

[6] R. Abe, M. Higashi, K. Sayama, Y. Abe, H. Sugihara, Photocatalytic Activity of R3MO7 and $\mathrm{R}_{2} \mathrm{Ti}_{2} \mathrm{O}_{7}$ ( $\mathrm{R}=\mathrm{Y}, \mathrm{Gd}$, La; $\mathrm{M}=\mathrm{Nb}, \mathrm{Ta}$ ) for Water Splitting into $\mathrm{H}_{2}$ and $\mathrm{O}_{2}$, J. Phys. Chem. B. 110 (2006) 22192226. https://doi.org/10.1021/jp0552933.

[7] J. Zhao, Q. He, B. Yao, Q. Zhang, T. Zhang, Q. Wang, X. Han, Hydrothermal synthesis of Y3NbO7 nanowires for the photocatalytic degradation of omeprazole sodium, Ceramics International. 41 (2015) 7669-7676. https://doi.org/10.1016/j.ceramint.2015.02.095.

[8] H. Yamamura, Electrical conductivity of the systems (YM)3NbO7 ( $\mathrm{M}=\mathrm{Ca}, \mathrm{Mg}$ ) and $\mathrm{Y} 3 \mathrm{NbMO7}(\mathrm{M}=\mathrm{Zr}$ and Ce), Solid State Ionics. 123 (1999) 279-285. https://doi.org/10.1016/S0167-2738(99)00098-3.

[9] D. Marrocchelli, P.A. Madden, S.T. Norberg, S. Hull, Cation composition effects on oxide conductivity in the Zr2Y2O7-Y3NbO7 system, Journal of Physics: Condensed Matter. 21 (2009) 405403.

[10] S.T. Norberg, I. Ahmed, S. Hull, D. Marrocchelli, P.A. Madden, Local structure and ionic conductivity in the Zr2Y2O7-Y3NbO7 system, J. Phys.: Condens. Matter. 21 (2009) 215401. https://doi.org/10.1088/0953-8984/21/21/215401.

[11] A. Chesnaud, M.-D. Braida, S. Estradé, F. Peiró, A. Tarancón, A. Morata, G. Dezanneau, Hightemperature anion and proton conduction in $\mathrm{RE} 3 \mathrm{NbO7}(\mathrm{RE}=\mathrm{La}, \mathrm{Gd}, \mathrm{Y}, \mathrm{Yb}, \mathrm{Lu}$ ) compounds, Journal of the European Ceramic Society. 35 (2015) 3051-3061. https://doi.org/10.1016/j.jeurceramsoc.2015.04.014.

[12] L. López-Conesa, J.M. Rebled, M.H. Chambrier, K. Boulahya, J.M. González-Calbet, M.D. Braida, G. Dezanneau, S. Estradé, F. Peiró, Local Structure of Rare Earth Niobates (RE3NbO7, RE = Y, Er, Yb, Lu) for Proton Conduction Applications ', Fuel Cells. 13 (2013) 29-33. https://doi.org/10.1002/fuce.201200136.

[13] K.-Y. Kim, A. Durand, J.-M. Heintz, A. Veillere, V. Jubera, Spectral evolution of Eu3+ doped Y3NbO7 niobate induced by temperature, Journal of Solid State Chemistry. 235 (2016) 169-174. https://doi.org/10.1016/j.jssc.2015.12.023.

[14] K.-Y. Kim, U.-C. Chung, B. Mutulet, F. Weill, A. Demourgues, J. Rossit, J.-M. Heintz, A. Veillere, V. Jubera, Tailoring the Composition of Eu3+-Doped Y3NbO7 Niobate: Structural Features and Luminescent Properties Induced by Spark Plasma Sintering, Inorganic Chemistry. 56 (2017) 44954503. https://doi.org/10.1021/acs.inorgchem.7b00088.

[15] J.G. Allpress, H.J. Rossell, Fluorite-related phases Ln3MO7, Ln= rare earth, Y, or Sc, M= Nb, Sb, or Ta. I. Crystal chemistry, Journal of Solid State Chemistry. 27 (1979) 105-114.

[16] H.J. Rossell, Fluorite-related phases Ln3MO7, Ln = rare earth, Y or Sc, M = Nb, Sb, or Ta, Journal of Solid State Chemistry. 27 (1979) 115-122. https://doi.org/10.1016/0022-4596(79)90150-6. 
[17] W.W. Barker, The systems Y2O3-Nb2O5 and Ho2O3-Nb2O5: the cubic solid-solution region, Journal of Materials Science Letters. 3 (1984) 492-494. https://doi.org/10.1007/BF00720980.

[18] K.P.F. Siqueira, J.C. Soares, E. Granado, E.M. Bittar, A.M. de Paula, R.L. Moreira, A. Dias, Synchrotron X-ray diffraction and Raman spectroscopy of Ln3NbO7 ( $\mathrm{Ln}=\mathrm{La}, \mathrm{Pr}, \mathrm{Nd}, \mathrm{Sm}$-Lu) ceramics obtained by molten-salt synthesis, Journal of Solid State Chemistry. 209 (2014) 63-68. https://doi.org/10.1016/j.jssc.2013.10.015.

[19] M.A. Subramanian, G. Aravamudan, G.V. Subba Rao, Oxide pyrochlores - A review, Progress in Solid State Chemistry. 15 (1983) 55-143. https://doi.org/10.1016/0079-6786(83)90001-8.

[20] A. Ikesue, T. Kinoshita, K. Kamata, K. Yoshida, Fabrication and Optical Properties of HighPerformance Polycrystalline Nd:YAG Ceramics for Solid-State Lasers, Journal of the American Ceramic Society. 78 (1995) 1033-1040. https://doi.org/10.1111/j.1151-2916.1995.tb08433.x.

[21] L. An, A. Ito, T. Goto, Fabrication of transparent Lu3NbO7 by spark plasma sintering, Materials Letters. 65 (2011) 3167-3169. https://doi.org/10.1016/j.matlet.2011.07.010.

[22] L. An, A. Ito, T. Goto, Transparent Lu3NbO7 bodies prepared by reactive spark plasma sintering and their optical and mechanical properties, Ceramics International. 39 (2013) 383-387. https://doi.org/10.1016/j.ceramint.2012.06.038.

[23] H. Kominami, M. Inoue, T. Inui, Formation of niobium double oxides by the glycothermal method, Catalysis Today. 16 (1993) 309-317. https://doi.org/10.1016/0920-5861(93)80070-H.

[24] T. Okubo, M. Kakihana, Low temperature synthesis of Y3NbO7 by polymerizable complex method: Utilization of a methanol-citric acid solution of $\mathrm{NbCl} 5$ as a novel niobium, (n.d.) 4 .

[25] R.D. Shannon, Revised effective ionic radii and systematic studies of interatomic distances in halides and chalcogenides, Acta Cryst A. 32 (1976) 751-767. https://doi.org/10.1107/S0567739476001551.

[26] C. Marlot, E. Barraud, S. Le Gallet, M. Eichhorn, F. Bernard, Synthesis of YAG nanopowder by the coprecipitation method: Influence of $\mathrm{pH}$ and study of the reaction mechanisms, Journal of Solid State Chemistry. 191 (2012) 114-120. https://doi.org/10.1016/j.jssc.2012.02.063.

[27] P. Palmero, R. Traverso, Co-Precipitation of YAG Powders for Transparent Materials: Effect of the Synthesis Parameters on Processing and Microstructure, Materials. 7 (2014) 7145-7156. https://doi.org/10.3390/ma7107145.

[28] R. Miida, F. Sato, M. Tanaka, H. Naito, H. Arashi, Locally Modulated Structures of Fluorite-Related Y2O3-Nb2O5 Solid Solutions, Journal of Applied Crystallography. 30 (1997) 272-279. https://doi.org/10.1107/S0021889896013283.

[29] J.-H. Lee, M. Yashima, M. Kakihana, M. Yoshimura, Phase Diagram and Oxygen-lon Conductivity in the Y2O3-Nb2O5 System, Journal of the American Ceramic Society. 81 (1998) 894-900.

[30] K.Y. Kim, A. Veillere, U.-C. Chung, V. Jubera, J.-M. Heintz, Spark plasma sintering and decomposition of the Y3NbO7:Eu phase, J Mater Sci. 53 (2018) 1731-1742. https://doi.org/10.1007/s10853-0171655-8.

[31] L. Wang, H. Kou, Y. Zeng, J. Li, Y. Pan, X. Sun, J. Guo, The effect of precipitant concentration on the formation procedure of yttrium aluminum garnet (YAG) phase, Ceramics International. 38 (2012) 3763-3771. https://doi.org/10.1016/j.ceramint.2012.01.022.

[32] M.N. Rahaman, Ceramic processing and sintering, in: Ceramic Processing and Sintering, Marcel Dekker Inc, New York, 2003: pp. 585-603.

[33] R.J. Brook, Treatise on materials science and technology. Vol. 9 : Ceramic fabrication processes, in: F.F.W. Wang ed, New York, 1976: pp. 331-364. 